\title{
114. On the Cosine Problem
}

\author{
By Miyoko Uchiyama, née Katayama, and Saburô Uchiyama \\ Department of Mathematics, Hokkaidô University, Sapporo, Japan
}

(Comm. by K. KUnUGI, M.J.A., Oct. 12, 1960)

1. Introduction. The main object of the present note is to establish the following theorem, which will answer in the affirmative to the cosine problem proposed by $\mathrm{S}$. Chowla in connexion with a question concerning zeta functions (cf. [1]):

Theorem 1. Let $K$ be an arbitrary positive number. Then there exists a natural number $n_{0}=n_{0}(K)$ such that for any $n \geqq n_{0}$ and any set of $n$ distinct positive integers $m_{1}, m_{2}, \cdots, m_{n}$ we have

$$
\min _{0 \leqq x<2 \pi}\left(\cos m_{1} x+\cos m_{2} x+\cdots+\cos m_{n} x\right)<-K .
$$

Here we may take

$$
n_{0}(K)=\max \left(2^{48},\left[8 K^{2}\right]^{3\left[256 K^{4}\right]}\right) \text {, }
$$

which is, of course, not the best possible.

As a simple generalization of Theorem 1 we can prove also that, given a real number $K>0$, there is an $n_{0}=n_{0}(K)$ such that for any $n \geqq n_{0}$ and any set of $n$ distinct positive integers $m_{1}, m_{2}, \cdots, m_{n}$ we have

$$
\min _{0 \leqq x<2 \pi} \sum_{j=1}^{n} \cos \left(m_{j} x+\omega_{j}\right)<-K,
$$

where $\omega_{1}, \omega_{2}, \cdots, \omega_{n}$ are arbitrary real numbers, and in particular,

$$
\min _{0 \leqq x<2 \pi} \sum_{j=1}^{n} \sin m_{j} x<-K, \quad \max _{0 \leqq x<2 \pi} \sum_{j=1}^{n} \sin m_{j} x>K .
$$

Thus Theorem 1 is a special case of the following

Theorem 2. Let $G$ be a locally compact connected abelian group. Given a real number $K>0$, we can find an $n_{0}=n_{0}(K)$ such that for any $n \geqq n_{0}$ and any set of $n$ distinct characters $\chi_{1}(x), \chi_{2}(x), \cdots, \chi_{n}(x)$ on $G$ we have

$$
\inf _{x \text { in } G} \operatorname{Re} \sum_{j=1}^{n} c_{j} \chi_{j}(x)<-K,
$$

where $c_{1}, c_{2}, \cdots, c_{n}$ are arbitrary complex numbers with $\left|c_{j}\right| \geqq 1(1 \leqq j$ $\leqq n)$.

For instance, if $\lambda_{1}, \lambda_{2}, \cdots, \lambda_{n}$ are arbitrary distinct positive real numbers, where $n \geqq n_{0}$, then we have

$$
\inf _{x \text { real }}\left(\cos \lambda_{1} x+\cos \lambda_{2} x+\cdots+\cos \lambda_{n} x\right)<-K .
$$

2. Some lemmas. In order to prove the theorems we appeal to a technique by $\mathrm{P}$. J. Cohen [2] developed in the investigation of a different problem, and so, to avoid ambiguity, we shall here reproduce some of his lemmas given in [2] with a slight modification. 
Let $X$ be the interval $[0,2 \pi]$. Let $C$ denote the space of all continuous functions defined on $X$ and $C_{0}$ be the subset of $C$ consisting of all functions with absolute values not greater than unity. If $\mu$ is a finite measure defined on $X$, we denote by $\|\mu\|$ the norm of $\mu$, i.e.

$$
\|\mu\|=\int_{x} d|\mu|(x) .
$$

Naturally, to such a measure $\mu$ there corresponds a linear functional $L$ on $C$ with the norm

$$
\|L\|=\sup \left|\int_{X} \phi(x) d \mu(x)\right|=\|\mu\|,
$$

where the supremum is taken over all $\phi(x)$ in $C_{0}$.

In what follows $\mu$ will be supposed to be a finite measure on $X$ such that $\|\mu\| \leqq M, M \geqq 1$.

Lemma 1. Let $g_{j}(x)(1 \leqq j \leqq r)$ be a set of functions in $C_{0}$ such that

$$
\int g_{j}(x) d \mu(x)=1 \quad(1 \leqq j \leqq r) .
$$

Then, if $r>2 M^{2}-1$, we have, for some pair $i<j$,

$$
\operatorname{Re} \int g_{i}(x) \bar{g}_{j}(x) d|\mu|(x)>\frac{1}{2 M} .
$$

Lemma 2. Let $\phi(x)$ and $g(x)$ be functions in $C_{0}$ satisfying the following conditions:

and

$$
\begin{array}{cl}
\int \phi(x) d \mu(x)=A & (A \geqq 1), \\
\left|\int g(x) d\right| \mu|(x)| \geqq \alpha & (0<\alpha<1),
\end{array}
$$

Then

$$
\int \phi(x) g(x) d \mu(x)=0
$$

$$
\|\mu\| \geqq A+\frac{\alpha^{2}}{4 A} .
$$

Lemma 3. Let $\phi(x)$ and $g_{j}(x)(1 \leqq j \leqq r)$ be functions in $C_{0}$ such that

and for all $i<j$,

$$
\begin{gathered}
\int \phi(x) d \mu(x)=A \quad(A \geqq 1), \\
\int g_{j}(x) d \mu(x)=1 \quad(1 \leqq j \leqq r),
\end{gathered}
$$

$$
\int \phi(x) g_{i}(x) \bar{g}_{j}(x) d \mu(x)=0 .
$$

Then, if $r>2 M^{2}-1$, we have 


$$
\|\mu\| \geqq A+\frac{1}{16 M^{3}} .
$$

By Lemma 1, for some pair $i<j$ we have

$$
\left|\int g_{i} \bar{g}_{j} d\right| \mu||>\frac{1}{2 M} .
$$

Put, in Lemma 2, $g=g_{i} \bar{g}_{j}$ with $\alpha=1 / 2 M$. Then

$$
\|\mu\| \geqq A+\frac{1}{16 A M^{2}} \geqq A+\frac{1}{16 M^{3}},
$$

since $A \leqq\|\mu\| \leqq M$.

Lemma 4. Under the hypotheses of Lemma 3, there exist constants $a, b_{j}, c_{i j}$ such that if

we have $|\psi(x)| \leqq 1$ on $X$ and

$$
\psi(x)=a \phi(x)+\sum_{j} b_{j} g_{j}(x)+\sum_{i<j} c_{i j} \phi(x) g_{i}(x) \bar{g}_{j}(x),
$$

$$
\int \psi(x) d \mu(x)=A+\frac{1}{16 M^{3}} \text {. }
$$

Let $V$ denote the linear subspace of $C$ generated by $\phi, g_{j}$ and $\phi g_{i} \bar{g}_{j}$. The measure $\mu$ induces a linear functional $L$ on $V$ with the norm $N$, say. The functional $L$ can be extended to a functional on the whole space $C$ with the same norm $N$, and the new functional is given by a measure satisfying the conditions of Lemma 3 . Hence

$$
N \geqq A+\frac{1}{16 M^{3}} .
$$

From this inequality the result follows at once.

Lemma 5. Let $E=\left\{m_{1}>m_{2}>\cdots>m_{n}\right\}$ be a set of $n$ distinct positive integers. If $r$ and $s$ are natural numbers satisfying

$$
r^{3 s} \leqq n,
$$

then there exist sets $F_{1}, \cdots, F_{s+1}, G_{1}, \cdots, G_{s}$ of positive integers with the following properties:

(a) $F_{1}=\left\{m_{1}\right\}$;

(b) for all $k, 1 \leqq k \leqq s, G_{k}=\left\{m_{k 1}>m_{k 2}>\cdots>m_{k r}\right\}$ is a subset of $E$ and $m+m_{k i}-m_{k j}$ is not contained in $E$ if $m$ is in $F_{k}$ and $i<j$;

(c) $F_{k+1}$ is the union of $F_{k}, G_{k}$ and all integers of the form $m+m_{k i}-m_{k j}$ with $m$ in $F_{k}, i<j$.

We denote by $h(k)$ the smallest integer $h$ such that $m \geqq m_{h}$ for all $m$ in $F_{k}$. Assume that the sets $F_{1}, \cdots, F_{k}, G_{1}, \cdots, G_{k-1}(k \geqq 1)$ have been chosen to satisfy the conditions (a), (b) and (c). We now define the set $G_{k}$. Set $m_{k 1}=m_{1}$. Suppose that $m_{k 1}, \cdots, m_{k t}(t \geqq 1)$ have been chosen so as to satisfy (b), where $m_{k i}=m_{j(i)}$ for $i \leqq t$. We then define $m_{k, t+1}=m_{j(t+1)}$, where $j(t+1)$ is the smallest number such that this choice of $m_{k, t+1}$ does not violate (b). The number of choices of $m_{k, t+1}<m_{k t}$ such that

$$
m+m_{k i}-m_{k, t+1} \in E
$$


for some $m$ in $F_{k}$ and $m_{k i}, i \leqq t$, does not exceed

$$
\frac{r(r-1)}{2} h(k) \text {. }
$$

Hence we find that

$$
j(t+1)-j(t) \leqq 1+\frac{r(r-1)}{2} h(k),
$$

and

$$
h(k+1)=j(r) \leqq r+\frac{r^{2}(r-1)}{2} h(k) \leqq r^{3} h(k),
$$

on defining the set $F_{k+1}$ by means of (c). Since $h(1)=1$, it follows that $h(k) \leqq r^{8(k-1)}$. Clearly the sets $F_{s}, G_{s}$, and hence $F_{s+1}$ can be constructed if $h(s+1) \leqq n$, or

$$
r^{3 s} \leqq n \text {. }
$$

That the sets $F_{k}$ and $G_{k}$ thus constructed contain only positive integers is obvious.

3. Proof of Theorem 1. There is no loss in generality in assuming that $K \geqq 1 / 2{ }^{*}$ Suppose now that the theorem were false. Then there would be a real number $K \geqq 1 / 2$ such that for arbitrarily large $n$ there exist $n$ distinct positive integers $m_{1}, \cdots, m_{n}$ for which the inequality

holds for all $x$ in $X$. Put

$$
\cos m_{1} x+\cdots+\cos m_{n} x \geqq-K
$$

$$
f(x)=K+\cos m_{1} x+\cdots+\cos m_{n} x=\frac{1}{2}\left(2 K+\sum_{j=1}^{n}\left(e^{i m_{j} x}+e^{-i m_{j} x}\right)\right) .
$$

Then $f(x) \geqq 0$ throughout on $X$. Now consider the finite, non-negative measure $\mu$ defined on $X$ by

$$
d \mu(x)=2 f(x) d x,
$$

where $d x$ is $1 / 2 \pi$ times the ordinary Lebesgue measure on $X$. We have

and for positive $m$,

$$
\|\mu\|=\int_{X} d \mu(x)=2 K \geqq 1,
$$

$$
\int_{X} e^{i m x} d \mu(x)= \begin{cases}1 & \text { if } m=m_{j} \text { for some } j, \\ 0 & \text { otherwise. }\end{cases}
$$

Without loss of generality we may suppose that $m_{1}>\cdots>m_{n}$. Put $r=\left[8 K^{2}\right]$ and $s$ be the largest integer satisfying (2). We construct functions $\phi_{k}(x)(1 \leqq k \leqq s+1)$, which are to be all in $C_{0}$, such that each $\phi_{k}(x)$ is a linear combination of $e^{i m x}$ with $m$ in $F_{k}$ and satisfies

$$
\int \phi_{k}(x) d \mu(x)=1+\frac{k-1}{128 K^{3}} \text {. }
$$

Take $\phi_{1}(x)=e^{i m_{1} x}$. If $\phi_{k}(x)(k \geqq 1)$ has already been defined, then

*) For $1 / 2 \geqq K>0$ we may take $n_{0}(K)=1$. 
by Lemmas 4 and 5 with

$$
g_{j}(x)=e^{i m x} \quad(1 \leqq j \leqq r)
$$

where $m=m_{k j}$ are in $G_{k}$, we can find a function $\psi(x)=\phi_{k+1}(x)$ in $C_{0}$ such that $\phi_{k+1}(x)$ is a linear combination of $e^{i m x}$ with $m$ in $F_{k+1}$ and

$$
\int \phi_{k+1} d \mu=1+\frac{k-1}{128 K^{3}}+\frac{1}{128 K^{3}}=1+\frac{k}{128 K^{3}} .
$$

Since we must always have

$$
\int \phi_{k} d \mu \leqq\|\mu\|=2 K
$$

it follows that

$$
1+\frac{s}{128 K^{3}} \leqq 2 K
$$

If $s=\left[256 K^{4}\right]$, this inequality cannot hold, so that necessarily

$$
\left[8 K^{2}\right]^{3\left[256 K^{4}\right]}>n \text {, }
$$

which is, however, certainly impossible when $n \geqq n_{0}$, where $n_{0}=n_{0}(K)$ is defined in (1). This completes the proof of Theorem 1.

4. Proof of Theorem 2. The passage of carrying our proof of Theorem 1 on that of Theorem 2 is substantially as indicated in [2, Lemmas $1^{\prime}$ and 5$]$, and we may omit the details.

\section{References}

[1] S. Chowla: The Riemann zeta and allied functions, Bull. Amer. Math. Soc., 58, 287-305 (1952).

[2] P. J. Cohen: On a conjecture of Littlewood and idempotent measures, Amer. J. Math., 82, 191-212 (1960). 